\title{
Ultra-low Noise Supercontinuum Generation with Flat-near Zero All Normal Dispersion Pure Silica Fiber at GHz Repetition Rate
}

\author{
Shreesha Rao D. S. ${ }^{1}$, Rasmus D. Engelsholm ${ }^{1}$, Iván B. Gonzalo ${ }^{1}$, Binbin Zhou ${ }^{1}$, \\ Patrick Bowen $^{2}$, Peter M. Moselund ${ }^{2}$, Morten Bache ${ }^{1}$, and Ole Bang ${ }^{1,2}$ \\ ${ }^{1}$ Department of Photonics Engineering, Technical University of Denmark, Ørsteds Plads, 2800, Lyngby, Denmark. \\ ${ }^{2}$ NKT Photonics A/S, Blokken 84, 3460 Birkerød, Denmark. \\ shdes@fotonik.dtu.dk
}

\begin{abstract}
A pure silica holey fiber with $\beta_{2}$ of $0.44 \mathrm{ps}^{2} / \mathrm{km}$ at $1.55 \mu \mathrm{m}$ and less than $1 \mathrm{ps}^{2} / \mathrm{km}^{2}$ from 1.3 to $1.75 \mu \mathrm{m}$ was engineered and drawn. It is numerically shown to generate a flat coherent spectrum, pumped by a $2 \mathrm{~kW}$ peak power, $250 \mathrm{fs}$ pulse propagating $20 \mathrm{~m}$.

OCIS codes: (320.6629) Supercontinuum generation, (060.2280) Fiber design and fabrication.
\end{abstract}

\section{Introduction}

All normal dispersion (ANDi) fibers with flat and close to zero dispersion generate flat and coherent supercontinuum (SC) when pumped by a sub-picosecond (sub-ps) pulse [1]. This is in contrast to SC generated by anomalous dispersion pumping (for soliton number, $\mathrm{N}>10$ ), where the generated spectrum shows pulse-to-pulse fluctuations even when pumped by a few hundred femtosecond (fs) pulse [2]. ANDi SC results in smooth spectral intensity and phase, preserves a single pulse in time domain which has lead to demonstration of sub-two cycle pulses from external recompression [3]. Spectra generated by these ANDi fibers enable applications that need flat and coherent SC such as optical coherence tomography (OCT), spectroscopy, and telecommunications.

\section{Fiber design and considerations}

The goal of this design is to obtain flat and coherent SC with high energy densities around 1.2 to $1.8 \mu \mathrm{m}$ using low peak power $\left(\mathrm{P}_{0}\right)$ fs pump at $1.55 \mu \mathrm{m}$. Flat dispersion ANDi designs made of soft glass [4], $\mathrm{GeO}_{2}$ doped [5] fibers, pumped at $1.55 \mu \mathrm{m}$ have been recently reported to generate coherent SC. Our pure silica microstructured fiber (MF) design has the advantage of being able to be drawn in already existing silica draw towers, without having to be doped. The designed fiber has a flat dispersion with $\beta_{2}$ of $0.44 \mathrm{ps}^{2} / \mathrm{km}$ at the pump and less than $1 \mathrm{ps}^{2} / \mathrm{km}$ in the entire wavelength region of interest (1.3 to $1.75 \mu \mathrm{m}$ ). The $\beta_{2}$ profile is plotted in Fig. 1(a) and 100x image of the drawn fiber is the inset.

\section{Results and discussion}

Coherent and smooth SC can be obtained from our fiber even by a sub-ps pump pulse. Since the fiber has flat and close to zero dispersion, new frequencies are generated coherently with the pump as broadening is dominated by self-phase modulation (SPM) and optical wave breaking (OWB). For sub-ps pulses in the fiber, broadening $\left(\Delta \omega_{c o h}\right)$ is independent of pulsewidth $\left(\mathrm{T}_{0}\right)$ and $\Delta \omega_{c o h} \propto\left(\gamma P_{0} / \beta_{2}\right)^{1 / 2}$. To achieve a flat spectrum with longer pulses, a longer fiber has to be used. In this case, the limiting factor for coherence is Raman amplified noise. The parametric Raman gain length, $\mathrm{L}_{R}^{\star}\left(=1 / g_{s}^{\star} P_{0}\right)$ becomes important when $\mathrm{L}_{R}^{\star}$ is greater than the characteristic length of broadening form SPM and OWB, since then the broadening would be noise seeded. The parametric Raman gain, $\mathrm{g}_{s}^{\star}$ is given by [1],

$$
g_{s}^{\star}=2 \gamma \operatorname{Re}(\sqrt{K(2 q-K)}) \text { with } \quad \text { (1) } \quad q=\left(1-f_{R}\right)+f_{R} \tilde{\chi}_{R}^{(3)}\left(-\Omega_{R}\right) \& \quad \text { (2) } \quad K=-\frac{\beta_{2} \Omega_{R}^{2}}{2 \gamma P_{0}}
$$

$\tilde{\chi}_{R}^{(3)}\left(-\Omega_{R}\right)$ is the complex Raman susceptibility of the Stokes frequency with maximum gain. Since the designed fiber has $\beta_{2}$ very close to zero, generated SC not only preferentially broadens towards the wavelength region with low values of dispersion but also has a large $\mathrm{L}_{R}^{\star}$ and thus avoids introducing Raman noise. This makes the fiber suitable to generate coherent SC even when pumped with pulse durations of several hundreds of fs. The fiber has a zero dispersion wavelength close to $1.8 \mu \mathrm{m}$ and the chosen fiber structure is such that the confinement loss above $1.8 \mu \mathrm{m}$ is high. This results in almost all the power to be in the normal dispersion region of the fiber when pumped at low $\mathrm{P}_{0}$. 


\subsection{Low peak power pumping}

Simulations were carried out by solving the scalar generalized nonlinear Schrödinger equation. With Origami 15 lasers from Onefive in mind we have used a pump pulse with full width at half maximum $\left(\mathrm{T}_{F W H M}\right)$ of $250 \mathrm{fs}$ and a low $\mathrm{P}_{0}$ of $2 \mathrm{~kW}$ at $1 \mathrm{GHz}$ repetition rate. The broadest spectrum was obtained at $7.5 \mathrm{~m}$ and further propagation upto $20 \mathrm{~m}$ resulted in a flat spectrum which is plotted in Fig. 1(c). $L_{R}^{\star}$ for $P_{0}$ of $2 \mathrm{~kW}$ is $0.75 \mathrm{~m}$ for the fiber. Since we propagate far longer than $\mathrm{L}_{R}^{\star}$, there is no guarantee that Raman noise is insignificant, but we can see from the Fig. 1 (b) that it is indeed the case, as the spectrum maintains excellent coherence. The generated SC covers all the wavelengths used in telecommunications and thus is perceived to be one of the main areas of application.
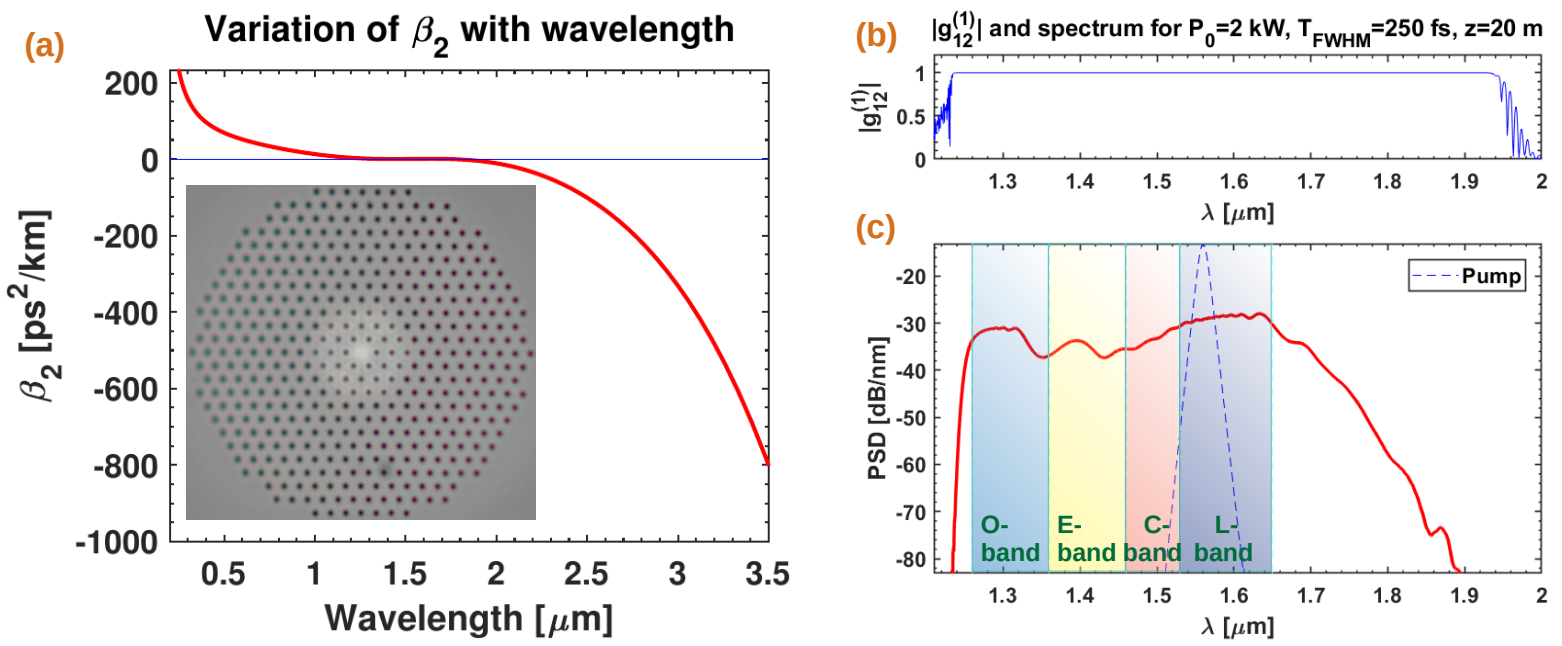

Fig. 1. (a) Dispersion profile of the fiber with the drawn fiber as inset. (b) Calculated degree of firstorder coherence from 30 single shot simulations with one photon per mode noise. (c) Simulated spectrum (red) for $\mathrm{T}_{F W H M}$ of $250 \mathrm{fs}$ and $\mathrm{P}_{0}$ of $2 \mathrm{~kW}$ of the pump propagation $20 \mathrm{~m}$ in the fiber.

\section{Conclusion}

A pure silica ANDi fiber with flat and close to zero dispersion was engineered and drawn. Supercontinuum generation in the fiber was studied numerically and is shown to generate flat spectrum when pumped by 250 fs pulse with $\mathrm{P}_{0}$ of 2 $\mathrm{kW}$ after propagating $20 \mathrm{~m}$ at GHz repetition rate, without the loss of coherence. The generated SC is well suited as a source for OCT as needed in [6] other than in telecommunications.

\section{Acknowledgments}

[1] European Union's Horizon 2020 research and innovation program under the Marie Skłodowska-Curie grant agreement No. 722380 (SUPUVIR). [2] Innovation Fund Denmark under the project J. No. 4107-00011A (ShapeOCT). [3] Det Frie Forskningsråd (DFF) (LOISE-4184-00532B).

\section{References}

1. Heidt, Alexander M., et al. "Limits of coherent supercontinuum generation in normal dispersion fibers." JOSA B 34.4 (2017): 764-775.

2. Dudley, John M., Goëry Genty, and Stéphane Coen. "Supercontinuum generation in photonic crystal fiber." Reviews of modern physics 78.4 (2006): 1135.

3. Heidt, Alexander M., et al. "High quality sub-two cycle pulses from compression of supercontinuum generated in all-normal dispersion photonic crystal fiber." Optics express 19.15 (2011): 13873-13879.

4. Klimczak, Mariusz, et al. "Coherent supercontinuum generation up to $2.3 \mu \mathrm{m}$ in all-solid soft-glass photonic crystal fibers with flat all-normal dispersion." Optics express 22.15 (2014): 18824-18832.

5. Tarnowski, Karol, et al. "Coherent supercontinuum generation up to $2.2 \mu \mathrm{m}$ in an all-normal dispersion microstructured silica fiber." Optics express 24.26 (2016): 30523-30536.

6. Maria, Michael, et al. "Q-switch-pumped supercontinuum for ultra-high resolution optical coherence tomography." Optics letters 42.22 (2017): 4744-4747. 